\title{
Arco axilar de Langer: serie de casos y revisión de la literatura
}

\author{
Langer's axillary arch: case series and literature review
}

\author{
Oscar Alejandro Bonilla-Sepúlveda (D)
}

MD, Msc Epidemiologia y Salud Pública, subespecialista en Mastología. Grupo de investigación en cáncer, Instituto de Cancerología, Clínica Las Américas. Profesor titular, posgrado de Ginecología y Obstetricia, UniRemington, Medellín, Colombia.

\section{Resumen}

Introducción. El arco de Langer es una entidad infrecuente, cuya prevalencia depende de la técnica quirúrgica utilizada y usualmente no se asocia con síntomas de compresión vascular o neuronal.

El objetivo de este estudio fue describir las características clínicas y morfológicas, y la proporción de síntomas de compresión neurovascular del arco de Langer, en mujeres con cáncer de mama llevadas a cirugía axilar.

Métodos. Estudio descriptivo del arco de Langer en mujeres con cáncer de mama, llevadas a cirugía axilar en el registro personal de un cirujano, en Medellín, Colombia, entre el 1 enero de 2017 y el 15 agosto de 2020. Se evaluaron características clínicas, morfológicas y síntomas de compresión neurovascular. Las variables categóricas se agruparon según su frecuencia como porcentajes, y para las variables continuas se calculó la mediana y su rango intercuartílico.

Resultados. Entre el 1 enero de 2017 y el 15 agosto de 2020 se realizaron 725 cirugías axilares, 479 biopsias de ganglio centinela y 246 linfadenectomías, encontrando 17 casos de arco de Langer, para una frecuencia de 2,3\%. Fue más frecuente encontrarlo en el curso de una linfadenectomía (n=11, 64,7 \%). En 15 (88,2 \%) casos se presentó riesgo de ocultamiento ganglionar y en 14 (82,3 \%) generó dificultad quirúrgica. No hubo casos con síntomas de compresión vascular o neuronal. En ningún caso se realizó el diagnostico imagenológico prequirúrgico. La conducta quirúrgica predominante fue sección, en 88,2 \%, sin presentar complicaciones quirúrgicas asociadas.

Discusión. Es importante para el cirujano el conocimiento del arco axilar como una variante anatómica de la axila, que puede ocultar los ganglios o dificultar la disección axilar, por lo que la conducta más usada es la sección.

Palabras clave: axila; músculo; biopsia del ganglio linfático centinela; escisión del ganglio linfático; neoplasias de la mama; Langer.

Fecha de recibido: 15/08/2020 - Fecha de aceptación: 14/10/2020 - Fecha de publicación en línea: 11/02/2021

Correspondencia: Oscar Alejandro Bonilla Sepúlveda, Centro Oncológico de Antioquía, Carrera 48 \# 46A sur-107, Envigado, Colombia. Teléfono 3221024 opción 7. Correo electrónico: mastologia.bonilla@gmail.com

Citar como: Bonilla-Sepúlveda OA. Arco axilar de Langer: serie de casos y revisión de la literatura. Rev Colomb Cir. 2021;36:268-74. https://doi.org/10.30944/20117582.646

Este es un artículo de acceso abierto bajo una Licencia Creative Commons - BY-NC-ND https://creativecommons.org/licenses/by-ncnd/4.0/deed.es 


\begin{abstract}
Introduction. Langer's arch is an infrequent entity, the prevalence of which depends on the surgical technique used and is usually not associated with symptoms of vascular or neuronal compression. The objective of this study was to describe the clinical and morphological characteristics, and the proportion of symptoms of neurovascular compression of Langer's arch, in women with breast cancer who underwent axillary surgery.
\end{abstract}

Methods. Descriptive study of Langer's arch in women with breast cancer, who underwent axillary surgery in the personal registry of a surgeon, in Medellín, Colombia, between January 1, 2017 and August 15, 2020. Clinical, morphological and clinical characteristics were evaluated for symptoms of neurovascular compression. Categorical variables were grouped according to their frequency as percentages, and the median and interquartile range were calculated for continuous variables.

Results. Between January 1, 2017 and August 15, 2020, 725 axillary surgeries, 479 sentinel node biopsies, and 246 lymphadenectomies were performed, finding 17 cases of Langer's arch, for a frequency of $2.3 \%$. It was more frequently found in the course of lymphadenectomy $(\mathrm{n}=11 ; 64.7 \%)$. In 15 (88.2\%) cases there was a risk of lymph node concealment and in $14(82.3 \%)$ it generated surgical difficulty. There were no cases with symptoms of vascular or neuronal compression. In no case was the pre-surgical imaging diagnosis made. The predominant surgical approach was section, in $88.2 \%$, without presenting associated surgical complications.

Discussion. Knowledge of the axillary arch as an anatomical variant of the axilla is important for the surgeon, which can hide the lymph nodes or make axillary dissection difficult, so the most commonly used approach is to cut it.

Keywords: axilla; muscle; sentinel lymph node biopsy; lymph node excision; breast neoplasms; Langer.

\section{Introducción}

El arco axilar fue identificado por primera vez por Alexander Ramsay en 1795 y publicado en 1812, pero fue hasta 1846 que Langer describió el músculo con mayor precisión, por lo que se conoció como "arco de Langer" ${ }^{1}$. El también llamado músculo axilo pectoral, o arco pectoro-dorsal, es una variación anatómica rara del músculo latísimo del dorso en la región axilar (figura 1).

Tiene una prevalencia del 1,7 a $7 \%$ en las disecciones axilares de cadáveres y de $6 \%$ en estudios de resonancia magnética nuclear (RMN) del hombro, pero de solo de $0,25 \%$ en cirugía ${ }^{2}$. No se han encontrado diferencias por género, pero se han descrito más casos en mujeres sometidas a tratamiento por cáncer de mama. Suele ser unilateral y predomina en el lado derecho para algunos autores, mientras que para otros su ubicación sería al azar; la bilateralidad es excepcional ${ }^{3}$.

Embriológicamente corresponde al remanente de una hoja más extensa de la musculatura asociada a la piel, en la unión a la grasa subcutánea, llamada "panniculus carnosus" ${ }^{4}$, altamente desarrollado en los mamíferos inferiores para permitir la movilidad funcional más amplia del brazo.
Se ha clasificado de diferentes maneras:

1. Según la estructura: Tipo I (muscular) y Tipo II (tendinosa), incluyendo cada uno cuatro subtipos diferentes, dependiendo de la inervación y del sitio de inserción ${ }^{5}$.

2. Según su origen: Categoría 1, la más frecuente, se origina desde el borde lateral del latísimo del dorso y se inserta en el aspecto postero superior del tendón pectoral mayor. Categoría 2 , arco incompleto, basada en el punto de in-

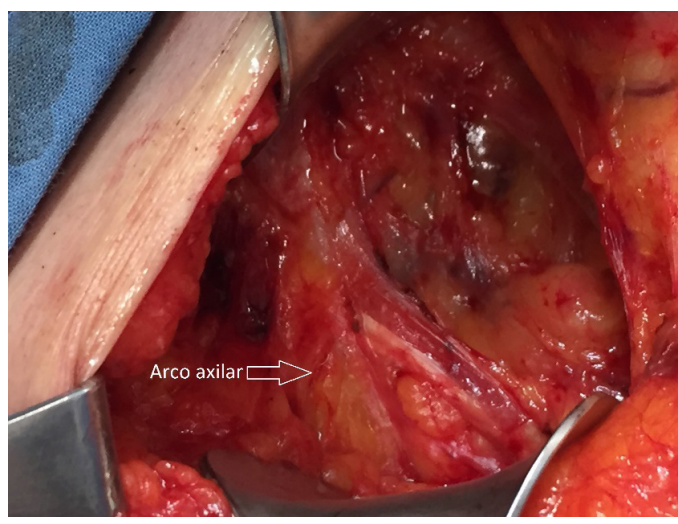

Figura 1. Arco de Langer durante disección axilar. 
serción distal, fue descrito por Testut en 1884, y puede insertarse en la fascia axilar, pectoral menor, bíceps braquial, coracobraquial, el proceso coracoide o el surco intertubercular ${ }^{6}$.

3. Según la forma: Tipo 1, surge como un musculo bien definido, horizontal, desde el borde lateral del latísimo del dorso. Tipo 2, consiste en fibras derivadas del dorsal ancho, que dejan el borde lateral del mismo para alcanzar inicialmente la axila, antes de cursar anterior y superiormente a su punto de inserción. Tipo 3 , es en forma de abanico y emerge del borde lateral del latísimo del dorso, para unir el arco tendinoso ${ }^{7}$.

Su contracción tiene poca importancia funcional, pero puede participar en el movimiento de flexión. Suele ser asintomático y su principal importancia es que impide una exposición adecuada del tejido linfograso, en especial en la linfadenectomía y en menor medida en la biopsia del ganglio centinela ${ }^{8}$, dificultando la disección de los ganglios linfáticos del grupo axilar lateral e inferior del nivel I de Berg, lo que da lugar a una linfadenectomía incompleta.

El objetivo de este artículo fue presentar una serie de casos de pacientes con arco axilar de Langer, y revisar sus características anatómicas y clínicas, así como sus implicaciones quirúrgicas.

\section{Métodos}

Diseño y población: Estudio descriptivo de serie de casos de pacientes con hallazgo intraoperatorio de la anomalía anatómica denominada arco axilar de Langer. Se incluyeron las pacientes con cáncer de mama diagnosticadas por biopsia con aguja gruesa del tumor, que fueron intervenidas con cirugía axilar, ya sea biopsia de ganglio centinela o linfadenectomía axilar, entre el $1^{\circ}$ de enero de 2017 y el 15 de agosto de 2020, en la ciudad de Medellín, Colombia.

Se realizó muestreo consecutivo de todos los casos con las características descritas, se construyó una base de datos prospectiva, donde se registró si un arco axilar era identificado durante el período de la muestra. Los datos fueron reco- lectados por el cirujano tratante.

Variables clínicas: Se tomaron en cuenta la edad, lateralidad axilar, el tipo de cirugía realizada en la mama y la axila, tamaño tumoral, numero de ganglios extirpados y afectados; presencia de síntomas compresivos vasculares como claudicación intermitente y síntomas neurales como dolor, parestesias, o debilidad en territorio del nervio mediano; complicaciones quirúrgicas, dificultad en la técnica dada por la necesidad de disecar el arco de Langer, y exploración vascular axilar. La determinación de ocultamiento ganglionar se registró cuando subyacente al arco de Langer había tejido linfograso asociado a los grupos ganglionares de Berg.

Análisis estadístico: Los datos ordinales y categóricos se resumieron en tablas de frecuencias y porcentajes; para las variables continuas se calcularon medidas de tendencia central y de dispersión, como media y desviación estándar o mediana y rango intercuartílico (RIC). Se calculó la proporción de arco de Langer, sus características anatómicas y quirúrgicas. La recolección de datos se hizo en una hoja Excel versión 9.0, y luego fueron analizados con el programa estadístico de distribución libre EPIDAT versión 3.1.

\section{Resultados}

En el periodo descrito se llevaron a cabo 725 disecciones axilares en mujeres con cáncer de mama, 479 correspondieron a biopsia de ganglio centinela y 246 a linfadenectomía axilar, encontrando 17 casos con arco de Langer, para una prevalencia global del 2,3\%. Se identificaron diferencias en la prevalencia para cada uno de los procedimientos, siendo del 1,2\% para la biopsia de ganglio centinela y 4,5\% para la linfadenectomía axilar.

La mediana de la edad fue de 60 años (RIC: 23) y la cirugía más efectuada fue la mastectomía, en $13(76,5 \%)$ pacientes (tabla 1). En ningún caso se realizó el diagnostico prequirúrgico y no se presentaron síntomas de compresión neurovascular.

El arco de Langer ocasionó dificultades para el cirujano en la disección axilar, durante la exploración vascular axilar, con ocultamiento ganglionar 
Tabla 1. Características clínicas de las pacientes con arco axilar de Langer.

\begin{tabular}{|c|c|c|}
\hline Característica & Frecuencia & $\%$ \\
\hline \multicolumn{3}{|l|}{ Lateralidad } \\
\hline Derecha & 5 & 29,4 \\
\hline Izquierda & 12 & 70,6 \\
\hline \multicolumn{3}{|l|}{ Cirugía mamaria } \\
\hline Mastectomía & 13 & 76,5 \\
\hline Cuadrantectomía & 4 & 23,5 \\
\hline \multicolumn{3}{|l|}{ Cirugía axilar } \\
\hline Ganglio centinela & 6 & 35,3 \\
\hline Linfadenectomía & 7 & 41,2 \\
\hline Ambos & 4 & 23,5 \\
\hline \multicolumn{3}{|c|}{ Diagnostico prequirúrgico } \\
\hline $\mathrm{Si}$ & 0 & 0 \\
\hline No & 17 & 100 \\
\hline \multicolumn{3}{|c|}{ Síntomas de compresión } \\
\hline \multicolumn{3}{|c|}{ Vascular } \\
\hline $\mathrm{Si}$ & 0 & 0 \\
\hline No & 17 & 100 \\
\hline \multicolumn{3}{|l|}{ Neural } \\
\hline $\mathrm{Si}$ & 0 & 0 \\
\hline No & 17 & 100 \\
\hline \multicolumn{3}{|c|}{ Complicaciones quirúrgicas } \\
\hline $\mathrm{Si}$ & 0 & 0 \\
\hline No & 17 & 100 \\
\hline \multicolumn{3}{|c|}{ Ocultamiento ganglionar } \\
\hline $\mathrm{Si}$ & 15 & 88,2 \\
\hline No & 2 & 11,8 \\
\hline \multicolumn{3}{|c|}{ Dificultad en la cirugía } \\
\hline $\mathrm{Si}$ & 14 & 82,3 \\
\hline No & 3 & 17,7 \\
\hline \multicolumn{3}{|c|}{ Intervención al arco de Langer } \\
\hline Corte o sección & 15 & 88,2 \\
\hline Preservación & 2 & 11,8 \\
\hline
\end{tabular}

en $15(88,2 \%)$ pacientes (figura 2) y demora en la disección en 14 (82,3\%). La conducta más frecuente fue la sección, en 15 (88,2 \%) casos; no se realizó sección en el trascurso de la biopsia de ganglio centinela, ya que no ameritaba una mayor exposición de la axila. No se presentaron complicaciones intraoperatorias.

Con relación a las características morfológicas (tabla 2), la longitud promedio fue de $5 \mathrm{~cm}$ (desviación estándar: 1,19) y el ancho promedio de 1,5 cm (desviación estándar: 0,35). La estructura más frecuente fue muscular $(64,7 \%)$; recordando que su origen es el musculo dorsal ancho, la inserción

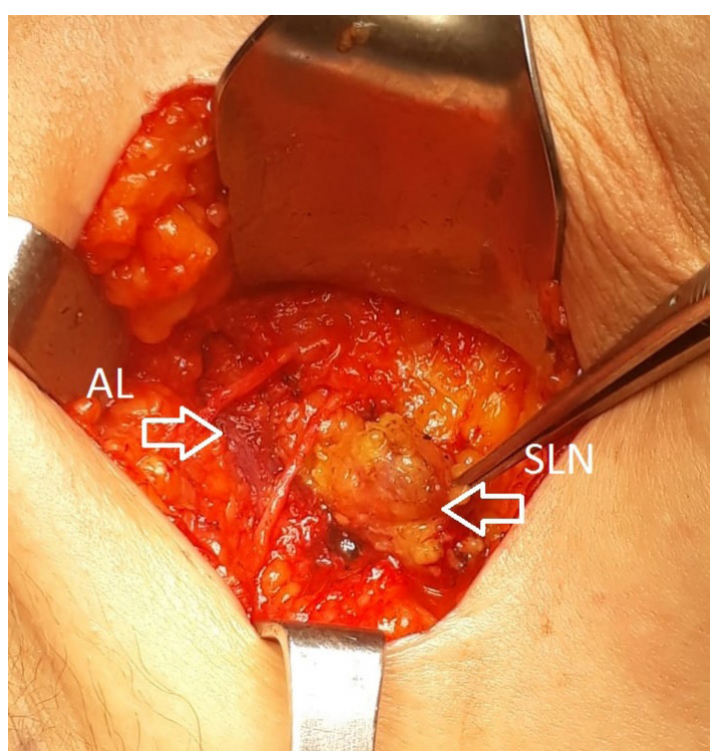

Figura 2. Arco axilar durante biopsia de ganglio centinela. AL: Arco de Langer. SLN: Ganglio centinela

se encontró con mayor frecuencia en el musculo pectoral mayor, en $13(76,5 \%)$ pacientes.

Esta es la serie de casos más grande reportada en la literatura mundial desde los años 90 y la primera en Latinoamérica (tabla 3), además una de las pocas que establece relación entre el arco de Langer y los síntomas de compresión neurovascular, el ocultamiento ganglionar y la conducta quirúrgica.

\section{Discusión}

El arco de Langer es una entidad infrecuente, con una prevalencia durante cirugía del 0,2 al $9,4 \%{ }^{2,9}$. En el presente estudio, entre 725 disecciones axilares practicadas por el mismo cirujano en un periodo de menos de 4 años, se calculó una frecuencia de $2,3 \%$, similar a la de otros estudios. Cuando se estima la frecuencia solo en los casos de linfadenectomía axilar, asciende a 4,5 \%, comparable a la encontrada en estudios con cadáveres de 7-8 \%, que demuestran una tasa de detección 2 a 3 veces mayor, diferencia que puede estar asociada a la mejor visualización de las estructuras axilares ${ }^{10}$.

La axila tiene forma de triángulo, conformada medial por los músculos pectoral mayor y serrato 
Tabla 2. Características morfológicas del arco axilar de Langer en las pacientes

\begin{tabular}{cccccccc}
\hline Paciente & $\begin{array}{c}\text { Tamaño del } \\
\text { tumor } \mathbf{( c m})\end{array}$ & $\begin{array}{c}\text { Total de } \\
\text { ganglios }\end{array}$ & $\begin{array}{c}\text { Ganglios } \\
\text { afectados }\end{array}$ & $\begin{array}{c}\text { Longitud } \\
(\mathbf{c m})\end{array}$ & $\begin{array}{c}\text { Ancho } \\
(\mathbf{c m})\end{array}$ & Estructura & Inserción \\
\hline 1 & 3 & 13 & 1 & 6 & 1,5 & Muscular & Coracoide \\
2 & 1,5 & 12 & 1 & 4 & 1,5 & Tendinoso & Pectoral mayor \\
3 & 7,5 & 16 & 7 & 6 & 2 & Tendinoso & Coracoide \\
4 & 2 & 1 & 0 & 4 & 1 & Muscular & Pectoral mayor \\
5 & 4 & 23 & 1 & 2,5 & 1,5 & Muscular & Coracoide \\
6 & 3,5 & 12 & 1 & 4 & 1,5 & Muscular & Pectoral mayor \\
7 & 6,5 & 17 & 1 & 3 & 1 & Muscular & Pectoral mayor \\
8 & 5 & 26 & 11 & 3,5 & 1 & Muscular & Pectoral mayor \\
9 & 5 & 26 & 11 & 5 & 1 & Tendinoso & Coracoide \\
10 & 7,5 & 12 & 0 & 4 & 2 & Muscular & Pectoral mayor \\
11 & 7 & 12 & 0 & 4 & 3 & Muscular & Pectoral mayor \\
12 & 4,5 & 15 & 3 & 5 & 2 & Muscular/ Tendinoso & Pectoral mayor \\
13 & 7 & 10 & 10 & 7 & 0,5 & Muscular & Pectoral mayor \\
14 & 12 & 2 & 0 & 8 & 1,5 & Muscular/ Tendinoso & Pectoral mayor \\
15 & 36 & 24 & 0 & 7 & 1,5 & Muscular & Pectoral mayor \\
16 & 0,3 & 12 & 2 & 6 & 1,5 & Muscular & Pectoral mayor \\
17 & 2 & 2 & 0 & 6 & 2 & Muscular/ Tendinoso & Pectoral mayor \\
\hline
\end{tabular}

Tabla 3. La incidencia de arco de Langer reportada en la literatura desde 1990.

\begin{tabular}{lcccc}
\hline Autor (año) & $\begin{array}{c}\text { Frecuencia de } \\
\text { arco de Langer }\end{array}$ & $\begin{array}{c}\text { Número de sujetos } \\
\text { evaluados }\end{array}$ & Porcentaje & Población \\
\hline Serpell - Baum (1991) & 4 & 2000 & 0,2 & Caucásico \\
Takafuji (1991) & 3 & 47 & 6,4 & Japonés \\
Clarys (1996) & 16 & 183 & 8,7 & Caucásico \\
Kalaycioglu (1998) & 1 & 60 & 1,7 & Caucásico \\
Miguel (2001) & 3 & 50 & 6 & Caucásico \\
Merida-Velasco (2003) & 3 & 32 & 9,4 & Caucásico \\
Turgut (2005) & 1 & 26 & 3,8 & Caucásico \\
Georgiev (2006) & 2 & 56 & 3,6 & Caucásico \\
Ortiz (2009) & 3 & 210 & 1,4 & Latino \\
\hline
\end{tabular}

anterior, superior por la vena axilar, y en la base por el musculo dorsal ancho, y contiene el paquete neurovascular axilar y los ganglios linfáticos ${ }^{10}$. El arco axilar de Langer se encuentra como un haz muscular triangular de base posterior, fusiforme, que mide entre 5 a $25 \mathrm{~mm}$ de espesor y tiene una longitud de 3,5 a $15 \mathrm{~cm}^{11}$.
En su forma clásica, se extiende del músculo latísimo del dorso, a la superficie profunda del músculo pectoral mayor, cruzando oblicuamente desde abajo hacia arriba y lateral ${ }^{12}$, y las variaciones más frecuentes en la inserción incluyen al pectoral menor o el proceso coracoide ${ }^{13}$. Posee dos porciones, una muscular, que acompaña el 
tendón del musculo pectoral mayor y se inserta en el surco intertubercular del humero, y una parte fascial, formada por bandas fibrosas, que se extiende hasta insertarse en el proceso coracoides, y atraviesa el pliegue posterior y medio de la axila, por delante del paquete neurovascular axilar ${ }^{2}$. Presenta muchas variaciones en origen, inserción, curso y tamaño.

En el presente estudio, el arco axilar tenía en promedio $5 \mathrm{~cm}$ de largo y $1,5 \mathrm{~cm}$ de ancho, discurrían de la porción tendinosa del músculo latísimo del dorso al borde inferolateral del musculo pectoral mayor, cruzando oblicuamente la fosa axilar. Se ha descrito la inserción a la parte anterior de la fascia profunda del músculo coracobraquial en el $76 \%$ y en la fascia profunda del músculo pectoral mayor en el $6 \%{ }^{14}$, mientras en el presente estudio se encontró la inserción en el coracoide en un $23,5 \%$ y en el musculo pectoral mayor en el $76,5 \%$.

La irrigación la proveen los vasos torácicos laterales, mientras que su inervación es variable $y$, dependiendo del origen embriológico y el sitio de inserción, puede venir del nervio pectoral menor, el nervio toracodorsal, nervio accesorio braquial cutáneo, nervio intercostobraquial, ramas penetrantes en el segundo y tercer nervios intercostales o nervio dorsal ancho ${ }^{15}$.

El arco axilar cubre un pequeño grupo de ganglios axilares laterales a medida que recorre la vena axilar. Su importancia para el cirujano está dada porque puede ocultar los ganglios linfáticos de nivel I en la disección axilar y llevar a una linfadenectomía incompleta ${ }^{10}$, o falta de migración del radiocoloide en la linfogamagrafía ${ }^{11}$, lo que produce subestadificación axilar, afectando negativamente la decisión de tratamiento sistémico adyuvante. No se conocen sus implicaciones en la recurrencia axilar en pacientes con cáncer.

En el presente estudio se encontró ocultamiento ganglionar en un $88,2 \%$ y dificultad en la técnica quirúrgica en $82,3 \%$, pero no hubo falla en la migración del radiocoloide en la linfogamagrafía.

Hay reportes que correlacionan la presencia del arco axilar y síntomas de compresión neuronal, como entumecimiento o dolor radiante ${ }^{14}$, que no se encontraron en el presente estudio. Se ha informado que el arco puede ser causa de atrapamiento de la vena axilar, con aparición de plenitud axilar y, más raramente, su compresión puede llevar a trombosis venosa o linfedema ${ }^{16}$. Incluso, puede ocasionar necrosis isquémica del latísimo del dorso en la reconstrucción mamaria, si el paquete toracodorsal es estirado o comprimido.

El examen físico con frecuencia es normal; ocasionalmente se puede encontrar una masa palpable dentro de la axila o una pérdida de la concavidad axilar. Algunos estudios han descrito la apariencia imagenológica del arco axilar, siendo inusual su diagnóstico preoperatorio ${ }^{14}$. En las mamografías, el músculo es visto solo en la proyección medio lateral oblicua, como una estructura parecida a una banda gruesa y suavemente ondulada, bilateral o unilateral de la axila, superpuesto al músculo pectoral mayor. En la tomografía computarizada o la resonancia magnética nuclear (RMN) es posible ver el curso exacto de este músculo a través de rastreo o reconstrucción multiplanar ${ }^{17}$. Es importante que los radiólogos conozcan de esta variante, debido a que puede tener relevancia preoperatoria para el cirujano. La RMN preoperatoria para la evaluación del arco no es una recomendación actual ${ }^{14}$, y en el presente estudio, en ningún caso se hizo un diagnostico preoperatorio.

La función de este músculo es insignificante, por lo que se puede cortar sin ningún déficit funcional ${ }^{10}$. Se recomiendan dos técnicas: cortar o disecar. La primera se prefiere por permitir mejor exposición y evitar el riesgo de compresión neurovascular, por eso fue la más frecuentemente utilizada en este grupo de pacientes. También es esencial durante la reconstrucción con colgajo miocutáneo dorsal ancho, para evitar el riesgo de compresión del pedículo escapular inferior y la isquemia ${ }^{18}$. La sección del arco de Langer requiere cuidado por el riesgo de lesión neurovascular, por su estrecha relación con el haz neurovascular toracodorsal, el plexo braquial y la vena axilar ${ }^{8}$, pero conservarlo lleva a los riesgos potenciales de compresión neurovascular. En la segunda técnica, el disecar el arco axilar pocos centímetros permite la disección neurovascular con seguridad, pero esta requiere práctica y prolonga significativamente el tiempo quirúrgico ${ }^{19}$. 


\section{Conclusión}

Es importante para el cirujano el conocimiento del arco axilar de Langer, como una variante anatómica de la axila, que puede ocultar ganglios o dificultar la disección axilar. Su prevalencia depende de la técnica quirúrgica usada y, no se encontró asociación con síntomas de compresión neuronal. La conducta quirúrgica más usada es la de cortarlo o seccionarlo.

\section{Cumplimiento de normas éticas}

Consentimiento informado: Investigación sin riesgo de acuerdo con la clasificación planteada en el Artículo 11 de la Resolución № 008430 de 1993 (expedida por el Ministerio de Salud de Colombia), y regido por las normas de la Declaración de Helsinki de 1975, modificada en el 2013. Se recolectó el consentimiento informado escrito y se preservó la confidencialidad de la información. El material fotográfico es exclusivo de la investigación.

Conflicto de interés: El autor declara no tener ningún conflicto de interés

Financiación: No hubo financiación externa ni fuentes de apoyo económico.

Contribución del autor: diseño; adquisición, análisis o interpretación de datos; redacción o revisión crítica y aprobación final del manuscrito: Oscar Alejandro Bonilla Sepúlveda.

\section{Referencias}

1. Langer C. Zur Anatomie Des Musculus Latissimus Dorsi. Oesterreichische Med Wochenschrift. 1846;15:454-58.

2. Bonastre V, Rodriguez-Niedenfuhr M, Choi D, Sañudo JR. Coexistence of a pectoralis quartus muscle and an unusual axillary arch: case report and review. Clin Anat. 2002;15:366-70. https://doi.org/10.1002/ca.10053

3. Kalaycioglu A, Gümüsalan Y, Ozan H. Anomalous insertional slip of latissimus dorsi muscle : arcus axillaris. Surg Radiol Anat. 1998;20:73-5. https://doi.org/10.1007/BF01628121

4. Gray H, Bannister LH, Berry M, eds. Gray's Anatomy; The Anatomical Basis of Medicine and Surgery. 38th edition. New York and London: Churchill Livingstone, 1995. p 782-3.

5. Takafuji T, Igarashi J, Kanbayashi T, Yokoyama T, Moriya A, Azuma S, Sato Y. The muscular arch of the axilla and its nerve supply in Japanese adults. Kaibogaku Zasshi. 1991;66:511-23.

6. Testut L. Les anomalies musculaires chez l'homme explique 'es par l'anatomie compare'e et leur importance en anthropologie. Masson, Paris, 1884. p 107-17. https://doi.org/10.5962/bhl.title.26341

7. Uzmansel D, Kurtoğlu Z, Kara A, Oztürk NC. Frequency, anatomical properties and innervation of axillary arch and its relation to the brachial plexus in human fetuses. Surg Radiol Anat. 2010;32:859-63. https://doi.org/10.1007/s00276-010-0687-4

8. Daniels IR, della Rovere GQ. The axillary arch of Langer - The most common muscular variation in the axilla. Breast Cancer Res Treat. 2000;59:77-80. https://doi.org/10.1023/A:1006367904056

9. Ortiz JI, Ramirez F, Petrosino P, Milano M, Arenas A, Castillo V. Arco axilar de Langer (músculo axilopectoral): Variante supernumeraria inusual del músculo latísimo del dorso. Reporte de tres casos. Int. J. Morphol. 2009;27:1209-12.

https://doi.org/10.4067/S0717-95022009000400039

10. Natsis K, Vlasis K, Totlis T, Paraskevas G, Noussios G, Skandalakis P, Koebke J: Abnormal muscles that may affect axillary lymphadenectomy: surgical anatomy. Breast Cancer Res Treat. 2009;120:77-82. https://doi.org/10.1007/s10549-009-0374-5

11. Kutiyanawala MA, Stotter A, Windle R. Anatomical variants during axillary dissection. Br J Surg. 1998;85:3934. https://doi.org/10.1046/j.1365-2168.1998.00612.x

12. Turgut HB, Peker T, Gülekon N, Anil A, Karaköse M. Axillopectoral muscle (Langer's muscle). Clin Anat. 2005;18:220-3. https://doi.org/10.1002/ca.20077

13. Jelev L, Georgiev GP, Surchev L. Axillary arch in human: common morphology and variety. Definition of "clinical" axillary arch and its classification. Ann Anat. 2007;189:473-81. https://doi.org/10.1016/j.aanat.2006.11.011

14. Guy MS, Sandhu SK, Gowdy JM, Cartier CC, Adams JH. MRI of the axillary arch muscle: prevalence, anatomic relations, and potential consequences. AJR Am J Roentgenol. 2011;196:W52-7. https://doi.org/10.2214/AJR.10.4380

15. Le Bouëdec G, Dauplat J, Guillot M, Vanneuville G. Le muscle pectoro-axillaire. J Chir (Paris). 1993;130:66-9.

16. Mérida-Velasco JR, Rodríguez-Vásquez JF, Mérida-Velasco JA, Sobrado-Pérez J, Jiménez-Collado J. Axillary arch: potential cause of neurovascolar compression syndrome. Clin Anat. 2003;16:514-9. https://doi.org/10.1002/ca.10143

17. Ko K, Han BK, Shin JH, Choe YH, Chung HW, Lee EH, Choi SJ. The axillopectoral muscle seen on mammography. Clin Radiol. 2006;61:625-9. https://doi.org/10.1016/j.crad.2006.03.015

18. Petrek J, Blackwood MM. Axillary dissection: current practice and technique. Curr Probl Surg. 1995;32:257332. https://doi.org/10.1002/ca.20077

19. Petrasek AJ, Semple JL, McCready DR. The surgical and oncologic significance of the axillary arch during axillary lymphadenectomy. Can J Surg. 1997;40:44-7. 